\title{
Efficient Person Identification by Fusion of Multiple Palmprint Representations
}

\author{
Abdallah Meraoumia ${ }^{1}$, Salim Chitroub ${ }^{1}$, and Ahmed Bouridane ${ }^{2}$ \\ 1 Signal and Image Processing Laboratory \\ Electronics and Computer Faculty, U.S.T.H.B. \\ P.O. Box 32, El-Alia, Bab-Ezzouar, 16111, Algiers, Algeria \\ 2 School of Computing, Engineering and Information Sciences \\ Northumbria University, Pandon Building \\ Newcastle upon Tyne, UK \\ Ameraoumia@gmail.com, S_chitroub@hotmail.com, Bouridane@qub.ac.uk
}

\begin{abstract}
The automatic person identification is a significant component in any security biometric system because of the challenges and the significant number of the applications that require a high safety. A biometric system based solely on one template (representation) is often not able to meet such desired performance requirements. Identification based on multiple representations represents a promising tendency. In this context, we propose here a multi-representation biometric system for person recognition using palm images and by integrating two different representations of the palmprint. Two ensembles of matchers that use two different feature representation schemes of the images are considered. The two different feature extraction methods are the block based 2D Discrete Cosine Transform (2D-DCT) and the phase information in 2D Discrete Fourier Transform (2D-DFT) that are complementing each other in terms of identification accuracy. Finally the two ensembles are combined and the fusion is applied at the matching-score level. Using the PolyU palmprint database, The results showed the effectiveness of the proposed multi-representation biometric system in terms of the recognition rate.
\end{abstract}

Keywords: Biometric, Palmprint, DCT, DFT, PCF, Data Fusion.

\section{Introduction}

The pronounced need for reliably determining or verifying the identity of person is becoming critical in our vastly interconnected information society [1. Traditional methods of establishing a person's identity, such as knowledge-based (passwords) and token-based (ID cards) mechanisms, are time-consuming, inefficient and expensive. In fact, a reliable identity management system is a critical component in several protected applications that render services to only legitimately enrolled users. However, theses surrogate representations of the identity can easily be lost, shared, manipulated or stolen thereby undermining the intended security. Biometrics offer a natural and reliable solution to the problem

A. Elmoataz et al. (Eds.): ICISP 2010, LNCS 6134, pp. 182-191, 2010.

(C) Springer-Verlag Berlin Heidelberg 2010 
of identity determination. Biometric authentication deals with recognition the identity of individuals by using their unique physical or behavioral characteristics that are inherent to the person.

However, every biometric technology has its merits and its limitations and no technology is the best for every application domain [2], 3], 4], 5]. Palmprint as a new biometric feature, compared to the hand-based biometric technologies such as fingerprint and hand geometry, has several advantages [6], 7], 8]. A simple biometric system has a sensor module, for acquiring the palmprint, a feature extraction module, for palmprint representation, and a matching module for decision making. Most of palmprint recognition systems are based on a single palmprint representation, and this fact can be considered as a bottleneck for performances. In fact, the performances of any biometric identification system are largely affected by the reliability of the method used for the feature extraction. Further, if the method used for the feature extraction is not efficient the resultant matching score computed by the matching module may not be reliable. An ideal palmprint recognition system should be based on the fusion of several palmprint representations. Such systems, known as multi-representations biometric systems, are expected to be more reliable due to the presence of multiple representations of evidence [6, 7], 8].

In this paper, we address the problem of information fusion by firstly building a multi-representations biometric system and then devising a scheme to integrate these representations. For that, we propose to use two different feature extraction methods. The extracted features are used as inputs of the matcher modules. The outputs of the matcher modules are combined using the concept of data fusion at score level. We propose to extract the palmprint feature using the 2D-DFT and the 2D-DCT. Thus, for each palm image, the two feature vectors are extracted and used for training two different matchers. The integration scheme is required to fuse the information presented by the individual representations.

The remainder of the paper is organized as follows. The proposed palmprint identification scheme is presented in section 2. The method used for extracting the Region Of Interest (ROI) is presented in section 3. The two feature extraction methods, 2D-DFT method and block based 2D-DCT method, are discussed in section 4. Sections 5 is devoted to describe the two matching modules, Sum of Absolute Differences (SAD) and Phase Correlation Function (PCF), and the score level fusion process for fusing the information presented by extracted features. The obtained results are evaluated and commented in section 6 . We conclude the present work in section 7 .

\section{Proposed Palmprint Identification Scheme}

Fig. 1 shows the block-diagram of the proposed palmprint identification system. The system is composed of three steps: image pre-processing, feature extraction and fusion. In the pre-processing module, the grey-scale image of the palm surface of the hand is segmented for extracting the Region Of Interest (ROI). In this system of multi-representations, the feature vectors are extracted 
independently from the unique ROI using the block based 2D-DCT and the phase information in 2D-DFT. Such extracted features are then compared to the enrolment templates, which are stored separately for each biometric trait. Based on the proximity of feature vector and template, by using SAD and PCF, each subsystem now computes its own matching score. After the score normalization of the matcher's outputs, these scores are finally fused into a total score, which is handed over to the decision module.

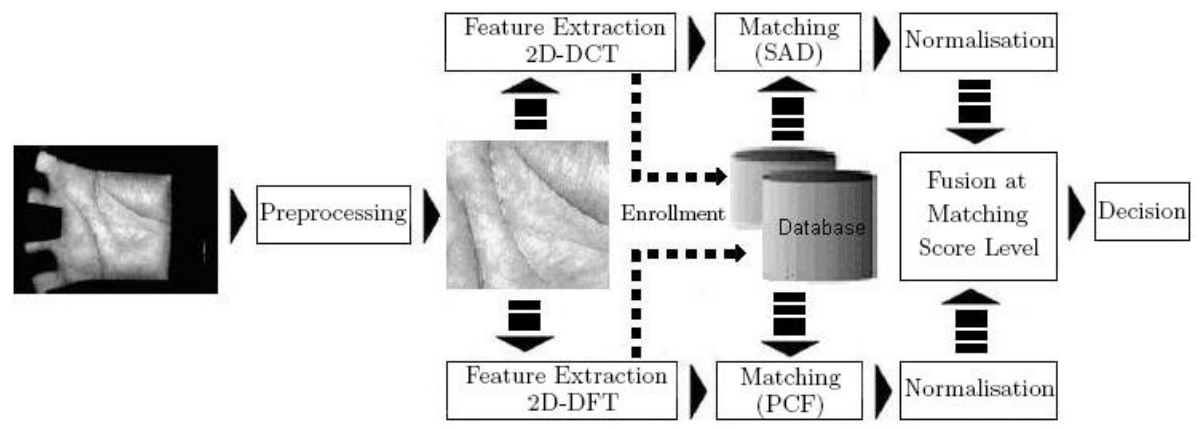

Fig. 1. Block-diagram of the multirepresentation palmprint identification system based on the fusion at the matching-score level

\section{Region Of Interest (ROI) Extraction}

After the image is captured, it is pre-processed to obtain only the area information of the hand. In the pre-processing phase, the tangent of the two holes, which are between the forefinger and the middle finger and between the ring finger and the little finger, are computed and used to arrange in a line the palmprint. With referencing to the locations of the gaps between fingers, the palm is duly rotated and the maximum palm area is then located. The central part of the image is then cropped to represent the whole palmprint. The ROI is then defined in a square shape and it is converted to a fixed size $(128 \times 128$ pixels $)$ so that all of the palmprints conform to a same size. The ROI extraction method applied in our system is based on the algorithm described in [5]. The basic steps to extract the ROI are summarized as follows: First, apply a low pass filter, such as Gaussian smoothing, to the original palmprint image. A threshold, $T_{p}$, is used to convert the filtered image to a binary image, then, the boundary tracking algorithm used to obtain the boundaries of the binary image. This boundary is processed to determine the points $F_{1}$ and $F_{2}$ for locating the ROI pattern and, based on the relevant points $\left(F_{1}\right.$ and $\left.F_{2}\right)$, the ROI pattern is located on the original image. Finally, the ROI is extracted. The pre-processing steps are shown in Fig. 2,

An example showing a sample of ROI patterns is given in Fig. 3. 


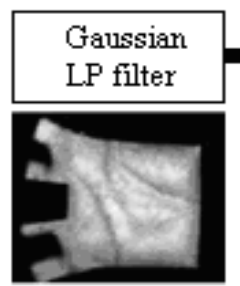

(a)

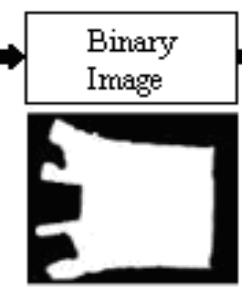

(b)

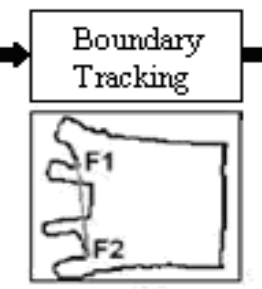

(c)

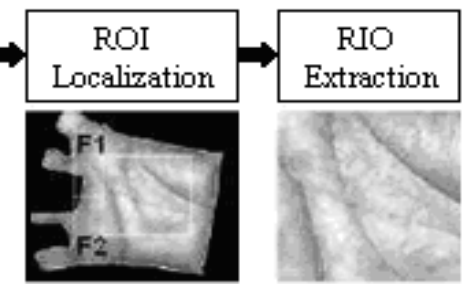

(d)

(e)

Fig. 2. Various steps in a typical region of interest extraction algorithm. (a) The filtered image, (b) The binary image, (c) The boundaries of the binary image and the points for locating the ROI pattern, (d) The central portion localization, and (e) The preprocessed result (ROI).
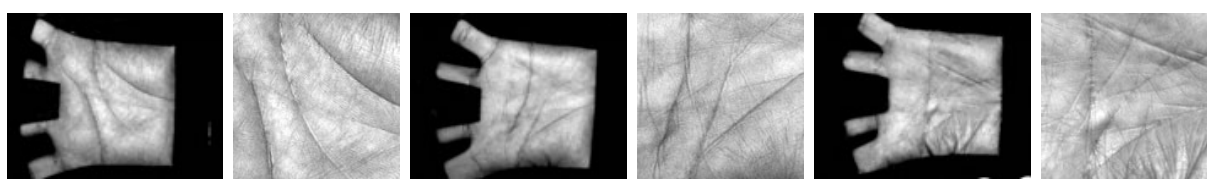

Fig. 3. Sample ROIs extracted from a palmprint

\section{Feature Extraction}

\subsection{Discrete Fourier Transform (DFT)}

The 2D-DFT of an input data with size $H \times H$ is defined as :

$$
F(u, v)=\frac{1}{H^{2}} \sum_{y=0}^{H-1} \sum_{x=0}^{H-1} f(x, y) \exp \left[2 \pi j\left(\frac{x u+y v}{H}\right)\right]
$$

Note that $f(x, y)$ is the image and is real, but $F(u, v), u, v=0,1,2, \ldots, H-1$, is the FT and is, in general, complex. Generally, $F$ is represented by its magnitude $(A)$ and phase $(\phi)$ rather that its real and imaginary parts, where:

$$
A(F)=\sqrt{\operatorname{Re}^{2}(F)+\operatorname{Im}^{2}(F)} \quad \phi(F)=\arctan \left[\frac{\operatorname{Im}(F)}{\operatorname{Re}(F)}\right]
$$

At the features-extraction stage the features are generated from the ROI subimage by $\phi(F)$. This feature-extraction technique has been used for matching module. It represents an efficient algorithm for palmprint recognition using the phase components in 2D-DFT of given images.

\subsection{Block Based Discrete Cosine Transform (DCT)}

With block based 2D-DCT, the image is analyzed on the basis of block per block. Given an image block, where $x ; y=0 ; 1 ; 2, \ldots . ., N-1$, we decompose it in terms 
of orthogonal 2D-DCT basis functions. The result is an $N \times N$ matrix $C(u ; v)$ containing DCT coefficients:

$$
C(u, v)=\alpha(u) \alpha(v) \sum_{y=0}^{N-1} \sum_{x=0}^{N-1} f(x, y) \cos \left[\frac{(2 y+1) v \pi}{2 N}\right] \cos \left[\frac{(2 x+1) u \pi}{2 N}\right]
$$

with $u, v=0,1,2, \ldots, N-1, \alpha(v)=\sqrt{1 / N}$ for $v=0$, and $\alpha(v)=\sqrt{2 / N}$ for $v=1, \ldots, N-1$. The coefficients are ordered according to a zigzag pattern. For a block located at $(b ; a)$, the baseline 2D-DCT feature vector is composed of :

$$
x_{1}=\left[\begin{array}{llll}
c_{0}^{(b, a)} & c_{1}^{(b, a)} & c_{2}^{(b, a)} & c_{3}^{(b, a)} \cdots \cdots c_{M-2}^{(b, a)} \\
& c_{M-1}^{(b, a)}
\end{array}\right]^{T}
$$

where $c_{n}^{(b, a)}, n \in[0, M]$ and $M=N \times N$,denotes the n-th 2D-DCT coefficient and $M$ is the number of coefficients. 2D-DCT-based features are also sensitive to changes in the illumination direction. Sanderson \& Paliwal [9] used a modified form of DCT feature extraction, termed DCT-mod2, which has been shown to be robust against illumination direction changes. In this paper, we introduce the DCT-mod2 feature extraction technique. For the DCT-mod2, the first three 2D-DCT coefficients (vector $x$ ) are replaced by their respective horizontal and vertical deltas, in order to reduce the effects of illumination direction changes, and thus form a feature vector representing a given block as follows [10]:

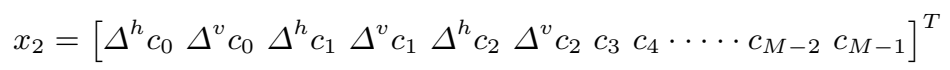

The n-th horizontal and vertical delta coefficient for block located at $(b ; a)$ are:

$$
\Delta^{h} C_{n}^{(b, a)}=\frac{\sum_{k=-K}^{K} k h_{k} c_{n}^{(b, a+k)}}{\sum_{k=-K}^{K} h_{k} k^{2}} \quad \Delta^{v} C_{n}^{(b, a)}=\frac{\sum_{k=-K}^{K} k h_{k} c_{n}^{(b+k, a)}}{\sum_{k=-K}^{K} h_{k} k^{2}}
$$

Where $h$ is a $2 K+1$ symmetric window vector. In this paper, we use $K=1$ and a rectangular window such as: $h=\left[\begin{array}{lll}1 & 1 & 1\end{array}\right]^{T}$.

\section{$5 \quad$ Matching Modules}

In the subsequent two feature-extraction modules, the phase information in 2DDFT and the block based 2D-DCT transform, are used for the extraction of the ROI sub-image features, while in the two matching modules the matching between the input feature and the templates from a database is performed. After the score normalization of the matchers outputs, fusion is applied. Tow different similarity/dissimilarity measures are used :

\subsection{Sum of Absolute Differences (SAD)}

For the features matching, the most popular criterion consists of minimizing the sum of the errors between the values of every pair of corresponding points 
between the input feature $F_{v}$ and the stored feature (template) $F_{r}$. In this context therefore, the objective function takes the form:

$$
E=\sum_{i=0}^{N-1} \sum_{j=0}^{N-1}\left|F_{v}(i, j)-F_{r}(i, j)\right|
$$

\subsection{Phase Correlation Function (PCF)}

Frequency domain palmprint matching is based on the 2D-DFT property that a translational displacement in the spatial domain corresponds to a linear phase shift in the frequency domain [11. Let two $N_{1} \times N_{2}$ images, the palmprint image to be verified (current) $f_{v}$ and the registered palmprint image (reference) $f_{r}$. Thus, assuming that $f_{v}$ and $f_{r}$ differ over a moving area, $\mathscr{A}$, only due to a translational displacement, $\left(\tau_{1} ; \tau_{2}\right)$.

$$
f_{v}\left(n_{1}, n_{2}\right)=f_{r}\left(n_{1}+\tau_{1}, n_{2}+\tau_{2}\right),\left(n_{1}, n_{2}\right) \in \mathscr{A}
$$

By taking the 2D-DFT of both sides :

$$
F_{v}\left(k_{1}, k_{2}\right)=F_{r}\left(k_{1}, k_{2}\right) e^{j\left(-k_{1} \tau_{1}-k_{2} \tau_{2}\right)}
$$

where $F_{v}$ and $F_{r}$ are the Fourier Transform (FT). If we define as the phase difference between the FT of $F_{v}$ and that of the $F_{r}$, then we obtain:

$$
e^{j \Delta \phi\left(k_{1}, k_{2}\right)}=e^{j\left[\phi_{v}\left(k_{1}, k_{2}\right)-\phi_{r}\left(k_{1}, k_{2}\right)\right]}=\frac{F_{v}\left(k_{1}, k_{2}\right)}{\left|F_{v}\left(k_{1}, k_{2}\right)\right|} \cdot \frac{F_{r}^{*}\left(k_{1}, k_{2}\right)}{\left|F_{r}^{*}\left(k_{1}, k_{2}\right)\right|}
$$

where $\phi_{v}$ and $\phi_{r}$ are the phase components of $F_{v}$ and $F_{r}$, respectively. If we define $C_{v, r}\left(n_{1}, n_{2}\right)$ as the inverse DFT of $e^{j \Delta \phi\left(k_{1}, k_{2}\right)}$, then we have:

$$
C_{v, r}\left(n_{1}, n_{2}\right)=\mathscr{F}^{-1}\left\{e^{j \Delta \phi\left(\left(k_{1}, k_{2}\right)\right.}\right\}=\mathscr{F}^{-1}\left\{e^{j \phi_{v}\left(k_{1}, k_{2}\right)}\right\} \otimes \mathscr{F}^{-1}\left\{e^{-j \phi_{r}\left(k_{1}, k_{2}\right)}\right\}
$$

where $\otimes$ is the $2 \mathrm{D}$ convolution operation. In other words, $C_{v, r}\left(n_{1}, n_{2}\right)$ is the crosscorrelation of the inverse 2D-DFT $\left(\mathscr{F}^{-1}=2 \mathrm{D}\right.$ IDFT $)$ of the phase components of $F_{v}$ and $F_{r}$. For this reason, $C_{v, r}\left(n_{1}, n_{2}\right)$ is known as the Phase Correlation Function (PCF) [12]. The importance of this function becomes apparent if it is rewritten in terms of the phase difference in equation (9):

$$
C_{v, r}\left(n_{1}, n_{2}\right)=\mathscr{F}^{-1}\left\{e^{j \Delta \phi\left(k_{1}, k_{2}\right)}\right\}=\delta\left(n_{1}-\tau_{1}, n_{2}-\tau_{2}\right)
$$

Thus, the phase correlation surface has distinctive impulses at $\left(\tau_{1} ; \tau_{2}\right)$. This observation is the basic idea behind the phase correlation matching. When two images are similar, their PCF gives a distinct sharp peak. When two images are not similar, the peak drops significantly. The elevation of the peak can be used as a good similarity measure for image matching. 


\subsection{Normalization and Fusion Modules}

Score level fusion is achieved by combining the matching scores calculated from different representations. Each representation has its matching module. Matching score is calculated with PCF-based matching and DCT-based matching. The two matching scores for the two feature vectors are normalized; i.e. it takes the values $[0,1]$, and combined using the strategy of the sum rule (based on their experimental results, the authors make the observation that the sum rule achieves the best performance [13]). In the decision stage, the best fusion score compared with the threshold of the decision, noted by $T_{E}$. When the best fusion score $\leq T_{E}$, the claimed identity is accepted; otherwise it is rejected.

\section{Experimental Results : Evaluation and Comments}

We have tested and evaluated our approach on (PolyU) palmprint database [14]. This database contains 100 classes. The size of the original Palmprint images is $384 \times 384$ pixels that have been captured with the resolution of $75 \mathrm{dpi}$. Twenty samples from each person were collected in two sessions. The palmprint images are aligned and the central part of the image, whose size is $128 \times 128$ pixels, is automatically segmented to represent the ROI of the palmprint. Three palmprint images of each palm were randomly selected for enrollment, and the remainder of 17 palmprint images is used as test images for recognition.

In the first experiment, the impostor distribution and the genuine distribution are generated by 1700 and 42075 comparisons for 100 persons, respectively. The Receiver Operating Curves (ROC) are plotted in Fig. 4, in which the Genuine Acceptance Rate (GAR) is plotted against False Acceptance Rate (FAR). For example, if only the 2D-DCT feature is used, we have GAR $=95.75 \%$ (FAR = $\mathrm{FRR}=4.25 \%$ ). In the case of using the 2D-DFT, the GAR is improved to 96.90 $\%(\mathrm{FAR}=\mathrm{FRR}=3.10 \%)$. By applying the fusion rule, the results are improved and they reach the rate of $97.95 \%(\mathrm{FAR}=\mathrm{FRR}=2.05 \%)$ for a database size equal to 100. Therefore; we can conclude from these results that an integration of 2D-DFT and 2D-DCT does result in an improvement of identification accuracy.

Other series of experiments are carried out using another palmprint database. Two experiments are done in order to test the identification performance of the system. We setup two registration databases for $\mathrm{N}=50$ and $\mathrm{N}=100$. No palmprint image of the testing database is contained in one of the registration databases.

In the first experimental with the database of fifty persons, and in order to obtain the performance characteristics such as the genuine and impostor distributions, the biometric feature of unknown user is presented to the system and the system will try to match the user with all stored template in the database. A genuine matching is defined as the matching between the palmprints from the same palm and an impostor matching is the matching between the palmprints from different palms. Therefore, a total of 21675 comparisons are performed, in which 850 comparisons are genuine matching and 20825 comparisons are impostor matching. The genuine and impostor distributions are plotted in Fig. 5, a 


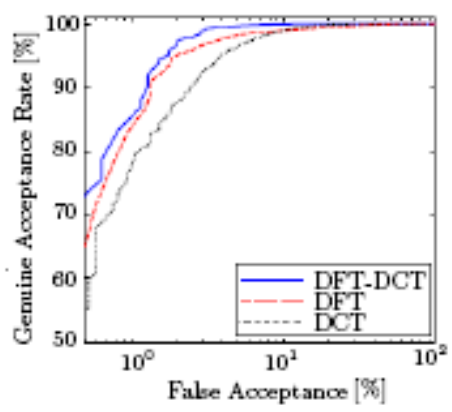

Fig. 4. Receiver operating curves for individual representations
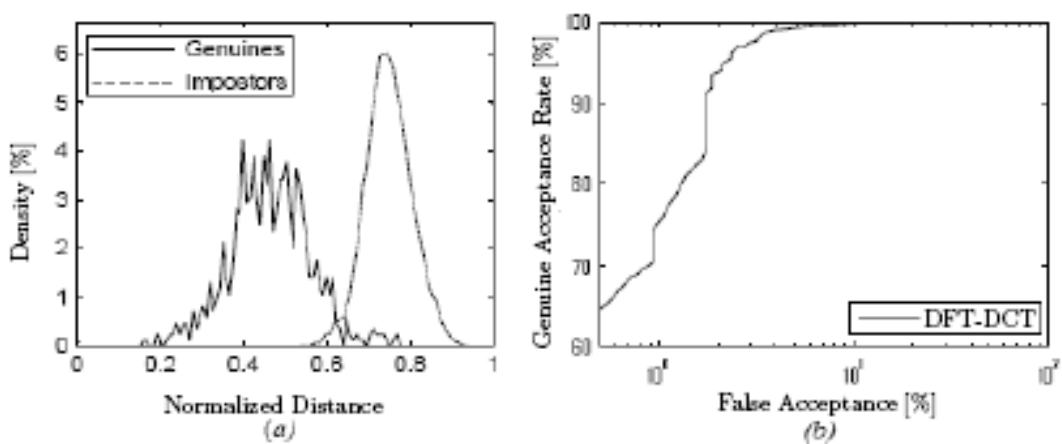

Fig. 5. Identification test results (50 Person's). (a) The genuine and impostor distribution, and (b) The receiver operating curves.
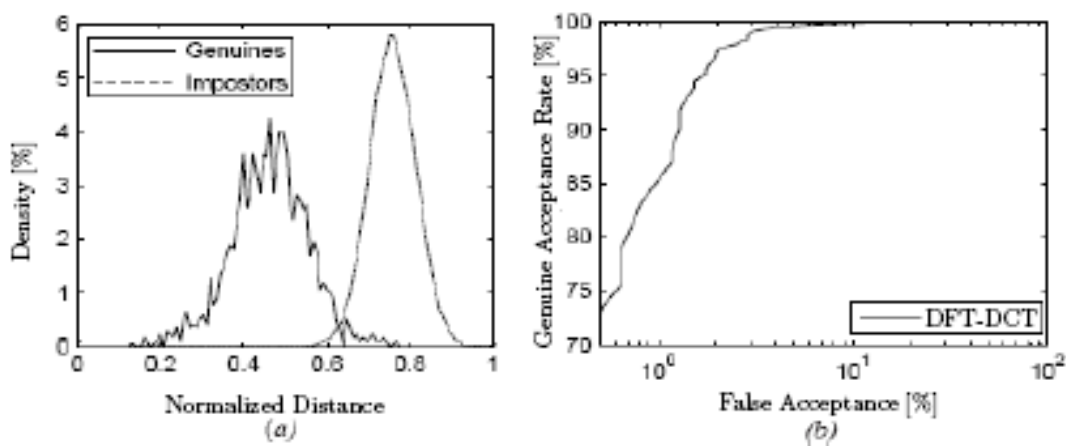

Fig. 6. Identification test results (100 Person's). (a) The genuine and impostor distribution, and (b) The receiver operating curves.

and the ROC curves, shown in Fig. 5.b, depicts the performance of the system. The described identification system can achieve the equality between FAR, False Rejection Rate (FRR) and Equal Error Rate (EER) that reaches the rate of 2.82 $\%$ at the decision threshold of $T_{E}=0.654$. A maximum of GAR is also reached 
with the rate of $97.18 \%$. Thus, we can conclude that the fusion of the two features has improved the results of the identification compared with results that have been obtained with only one feature.

In the second experimental with the database of one hundred persons, the distributions of the impostor and the genuine are generated by 1700 and 42075 comparisons, respectively. Fig. 6] presents the results of the identification and Fig. 6, b shows the corresponding ROC. As in the first experimental, the identification system can achieve the equality between FAR, FRR and EER with the rate of $2.25 \%$ for $T_{E}=0.656$ and a maximum of GAR is reached at the rate of $97.75 \%$. These experimental results show that the proposed methods reach a highest identification rate compared with other methods developed in the literature 15 and 16 .

\section{Conclusion}

A biometric system that is based only on a single biometric characteristic may not always be able to achieve the desired performance. Biometric techniques based on the multi-representations, which combine multiple representations in identification process, can be used to overcome the limitations of the single biometric system. Integration of multi-representations of the biometrics for an identification system can improve the identification accuracy. We have developed a decision fusion scheme that integrates two different representations that complement each other, which are the block based 2D-DCT and the phase information in 2D-DFT. A fusion scheme exploits all the information of the two representations via its decision rule. In order to demonstrate the efficiency of our proposed fusion scheme, the (PolyU) palmprint database for testing and evaluating the method is used. The obtained results show that the proposed fusion scheme overcomes many of the limitations of both individual systems, DCT based identification system and DFT based identification system. They demonstrate that it performs very well and it meets the accuracy requirements.

\section{References}

1. Jain, A.K., Bolle, R., Pankanti, S.: Biometrics Personal Identification in Networked Society. The Kluwer International Series in Engineering and Computer Science, USA (2002)

2. Ross, A.A., Nandakumar, K., Jain, A.K.: Handbook of Multibiometrics. Springer Science+Business Media, LLC (2006)

3. Ross, A., Jain, A.K.: Information Fusion in Biometrics. Pattern Recognition Letters 24(13), 2115-2125 (2003)

4. Ma, Y., Cukic, B., Singh, H.: A Classification Approach to Multi-Biometric Score Fusion. In: Kanade, T., Jain, A., Ratha, N.K. (eds.) AVBPA 2005. LNCS, vol. 3546, pp. 484-493. Springer, Heidelberg (2005)

5. Zhang, D., Kong, W., You, J., Wong, M.: On-line Palmprint Identification. IEEE Trabsactions on Pattern Analysis and Machine Intelligence 25(9), 1041-1050 (2003) 
6. Kong, W.K., Zhang, D.: Palmprint Texture Analysis Based on Low-Resolution Images for Personal Authentication. In: The 16th International Conference on Pattern Recognition, August 2002, vol. 3, pp. 807-810 (2002)

7. Nanni, L., Lumini, A.: Ensemble of Multiple Palmprint Representation. Expert Systems with Applications (2008)

8. Kumar, A., Wong, D.C.M., Shen, H.C., Jain, A.K.: Personal Verification Using Palmprint and Hand Geometry Biometrics. In: The Fourth International Conference on Audio and Video-Based Biometric Personal Authentication (June 2003)

9. Sanderson, C., Paliwal, K.K.: Polynomial Features for Robust Face Authentication. In: Proceedings of International Conference on Image Processing, Rochester, New York, vol. 3, pp. 997-1000 (2002)

10. Sanderson, C., Paliwal, K.K.: Fast feature extraction method for robust face verification. Electronics Letters 38(25) (2002)

11. Kumar, A., Mahalanobis, A., Juday, R.: Correlation Pattern Recognition. Cambridge University Press, New York (2005)

12. Ito, K., Nakajima, H., Kobayashi, K., Aoki, T., Higuchi, T.: A fingerprint matching algorithm using phase-only correlation. IEICE Transactions on Fundamentals E87A(3) (2004)

13. Bubeck, U.M.: Multibiometric Authentication-An Overview of Recent Developments-. Term Project CS574 Spring, San Diego State University (2003)

14. The Hong Kong Polytechnic University, PolyU Palmprint Database, http://www.Comp.polyu.edu.hk/biometrics

15. Pang, Y.H., Teoh Beng Jin, A., Ngo, D., Ling, C.: Palmprint Authentication System Using Wavelet based Pseudo Zernike Moments Features. Inter. Journal of The Computer, the Internet and Management 13(2), 13-26 (2005)

16. Hennings, P., Kumar, A.: Palmprint Recognition Using Correlation Filter Classifiers. IEEE Transactions on Circuits and Systems for Video Technology (2004) 